Supporting Information for:

\title{
Chromate Effect on Iodate Incorporation into Calcite
}

Sarah A. Saslow ${ }^{1 *}$, Sebastien N. Kerisit ${ }^{2}$, Tamas Varga ${ }^{3}$, Kayla C. Johnson ${ }^{1}$, Nancy M. Avalos ${ }^{1}$, Amanda R. Lawter ${ }^{1}$, Nikolla P. Qafoku ${ }^{1}$

${ }^{1}$ Energy and Environment Directorate, Earth Systems Science Division, Pacific Northwest National Laboratory, Richland, WA 99354, USA

${ }_{2}^{2}$ Physical and Computational Sciences Directorate, Physical Sciences Division, Pacific Northwest National Laboratory, Richland, WA 99354, USA

${ }^{3}$ Environmental Molecular Sciences Laboratory, Earth and Biological Sciences Directorate, Pacific Northwest National Laboratory, Richland, WA, 99354, USA

*Corresponding author: Sarah A. Saslow, Pacific Northwest National Laboratory, 902 Battelle Blvd., PO Box 999, P7-54, Richland, WA 99352, USA. Telephone: (509)-371-7175. Email address: sarah.saslow@pnnl.gov.

Summary of Supporting Information:

7 pages (including cover page), 5 Figures and 1 Table 

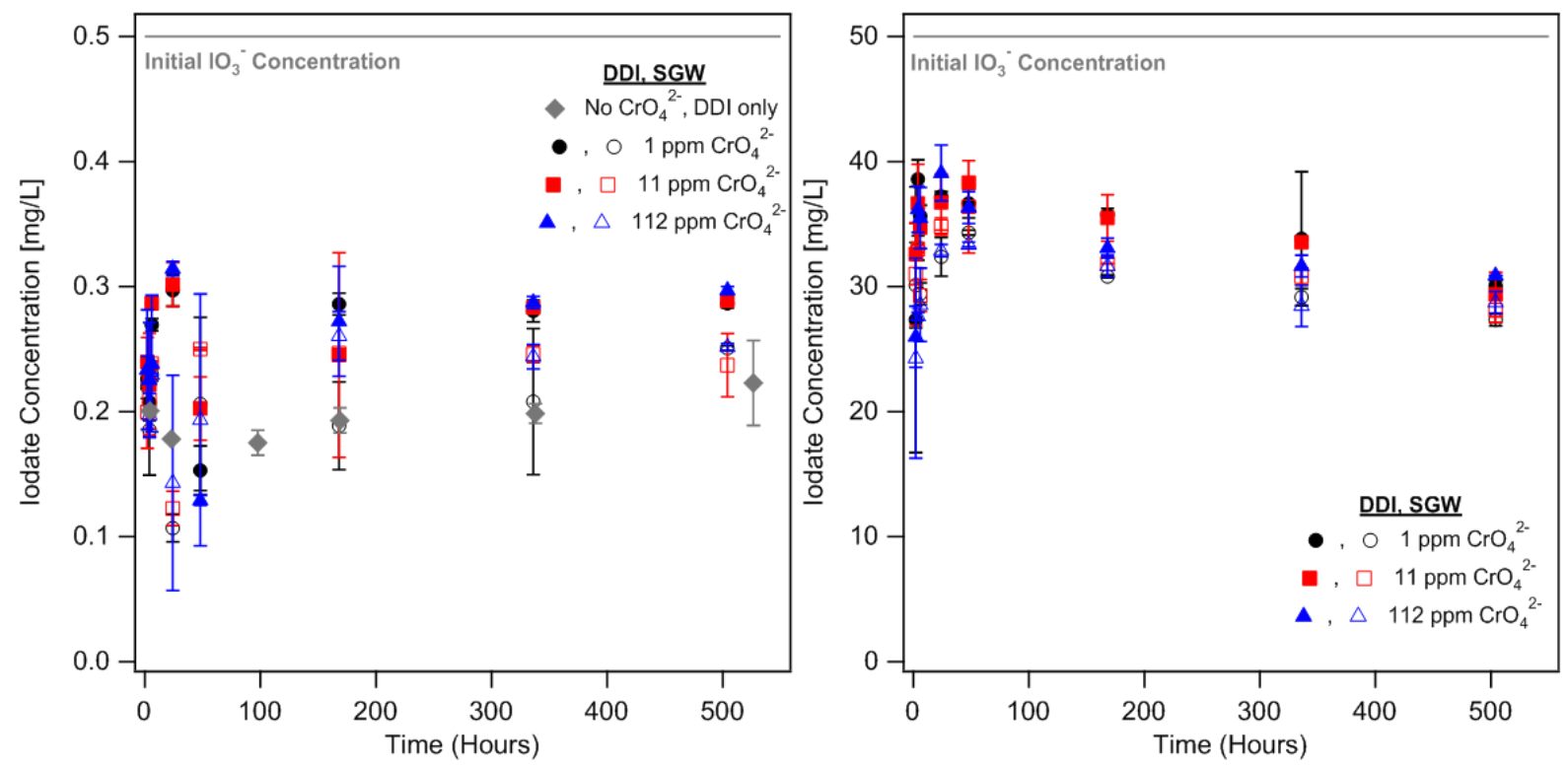

Figure S1. Iodate concentration in the aqueous phase as a function of time. Initial iodate concentration in solution is $0.5 \mathrm{ppm}$ (left) and $50 \mathrm{ppm}$ (right). Removal from DDI solutions are represented by filled markers and empty markers represent SGW samples. Error bars indicate the range in removal as determined from duplicate experiments.
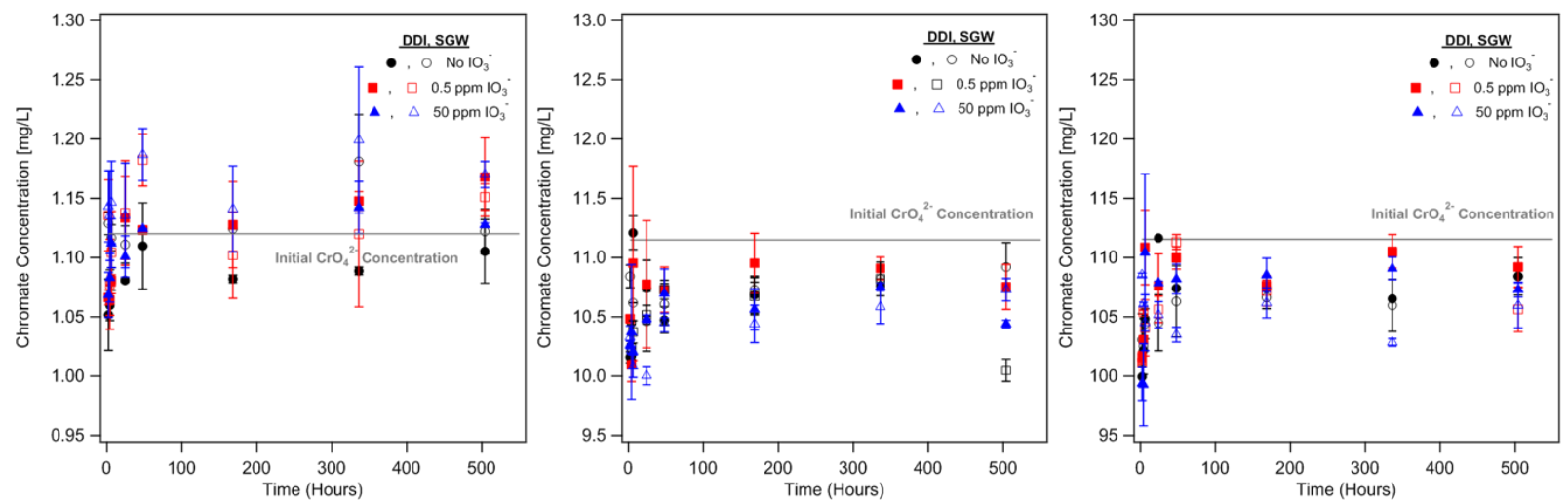

Figure S2. Chromate concentration in the aqueous phase as a function of time. Initial chromate concentration in solution is $1.1 \mathrm{ppm}$ (left), $11 \mathrm{ppm}$ (middle), and $112 \mathrm{ppm}$ (right). Removal from DDI solutions are represented by filled markers and empty markers represent results from SGW solutions. Error bars indicate the range in removal as determined from duplicate experiments. 
AIMD and EXAFS Fitting Method Details. Plane-wave density functional theory (DFT) calculations were performed with VASP (Vienna Ab-initio Simulation Package) ${ }^{1-6}$ using the projector augmented-wave (PAW) approach and the generalized gradient approximation exchange-correlation functional of Perdew, Burke, and Ernzerhof (PBE) ${ }^{7}, 8$ with Grimme dispersion corrections (G). ${ }^{9}$ The PBE PAW potentials were obtained from the VASP database for iodine (46), chromium (18), calcium (12), sodium (4), oxygen (2), carbon (2), and hydrogen (0) with the number of core electrons shown in parentheses.

A constant-pressure energy minimization was first performed to determine the optimized crystal structure of calcite at the $\mathrm{PBE}+\mathrm{G}$ level of approximation and the optimized unit cell was then scaled to a $2 \times 2 \times 1$ supercell size. Three series of energy minimizations were performed with the calcite supercell. In the first series, one $\mathrm{CO}_{3}{ }^{2-}$ was substituted by one $\mathrm{IO}_{3}{ }^{-}$and the net charge thus introduced was compensated by replacing one $\mathrm{Ca}^{2+}$ cation either by $\mathrm{Na}^{+}$or $\mathrm{H}^{+}$. Multiple initial positions of $\mathrm{Na}^{+}$and $\mathrm{H}^{+}$relative to $\mathrm{IO}_{3}{ }^{-}$were considered and these calculations were reported in a previous publication. ${ }^{10}$ In the second series, one $\mathrm{CO}_{3}{ }^{2-}$ was replaced by one $\mathrm{CrO}_{4}{ }^{2-}$. Because this substitution does not introduce a net charge, a charge compensation scheme was not needed and this series consisted of a single calculation. In the third series, one $\mathrm{CO}_{3}{ }^{2-}$ was substituted by one $\mathrm{CrO}_{4}{ }^{2-}$ starting from two important configurations from the first series (one with $\mathrm{Na}^{+}$and the other with $\mathrm{H}^{+}$as the charge compensating species), as determined from their significant contributions to linear combination fits to the K-edge spectrum of iodate incorporated in calcite, ${ }^{10}$ whereby $\mathrm{CrO}_{4}{ }^{2-}$ was placed in the first, second, or third nearest-neighbor carbonate positions of the iodate ion. The energy minimizations used a plane-wave cutoff energy of $650 \mathrm{eV}$ and a $3 \times 3 \times 1 \mathrm{k}$-point mesh.

NVT (constant number of particles, constant volume, and constant temperature) AIMD simulations were the performed at $8 \mathrm{~K}$ for key configurations in the first series, as reported in a previous publication, ${ }^{10}$ the sole configuration in the second series, and all six configurations of the third series. All the AIMD simulations used a slightly reduced plane-wave cutoff energy of 600 $\mathrm{eV}$ and were performed at the $\Gamma$ point $(1 \times 1 \times 1 k$-point mesh) with a 0.5 fs integration time step. The AIMD simulations were run for 7 ps whereby the atomic velocities were scaled to the target temperature every 12.5 fs for the first picosecond of simulation. Configurations were collected from each simulation at $50 \mathrm{fs}$ intervals for the last 5 ps to generate a pool of 100 configurations.

For each configuration, a cluster with a radius of $10 \AA$ centered on the iodine or chromium atom was then generated to calculate all scattering paths with effective distances less than the cluster radius for an iodine or chromium K-core hole of the central atom using FEFF9. ${ }^{11-13}$ The spectra of all configurations were averaged for comparison with experiment. The $S_{0}{ }^{2}$ parameters ( 0.878 and 0.955 for iodine and chromium, respectively) calculated by FEFF9 were used in all calculations. The Fourier transform (FT) was applied to the averaged EXAFS spectra using IFEFFIT. ${ }^{14}$ The calculated spectra were used as standards in linear combination fits of the experimental spectra to determine the local coordination environment around iodine and chromium. 
Table S1. Linear combination fits. Reciprocal-space fits to the EXAFS data collected in this work (50 ppm iodate and $100 \mathrm{ppm}$ chromate in DDI) and to that of Kerisit et al. ${ }^{10}$ were performed over the $k$ range $3.5 \AA^{-1} \leq k \leq 16.3 \AA^{-1}$. Bulk incorporation configurations are labeled as $\mathrm{B} X$, where $X$ is a digit used to differentiate between the different positions of the charge compensating species, and surface incorporation configurations are labeled as $\mathrm{S}_{\mathrm{d}}^{X}$, where $X$ represents the surface atomic layer iodate is incorporated in and d indicates that the surface is dry. In each case, the label is preceded by either $\mathrm{Na}$ or $\mathrm{H}$ to indicate the charge compensating species. Graphical representations of the incorporation configurations and associated details of the calculations can be found in Kerisit et al. ${ }^{10}$

\begin{tabular}{lcccccccccccc}
\hline Data & $T(\mathrm{~K})$ & $k$ weight & reduced $\chi^{2}$ & $\mathrm{NaB} 1$ & $\mathrm{Na} \mathrm{B} 2$ & $\mathrm{Na} \mathrm{B} 3$ & $\mathrm{Na} \mathrm{B} 4$ & $\mathrm{H} \mathrm{B} 1$ & $\mathrm{H} \mathrm{B}_{2}$ & $\mathrm{H} \mathrm{B3}$ & $\mathrm{Na} \mathrm{S}$ & $\mathrm{H} \mathrm{S}_{\mathrm{d}}^{1}$ \\
\hline This work & 8 & 3 & 2.045 & 0.07 & - & - & - & - & - & 0.33 & - & 0.60 \\
Kerisit et al. $^{10}$ & 8 & 3 & 1.581 & - & 0.13 & 0.03 & - & 0.01 & 0.02 & 0.31 & 0.08 & 0.43 \\
\hline
\end{tabular}



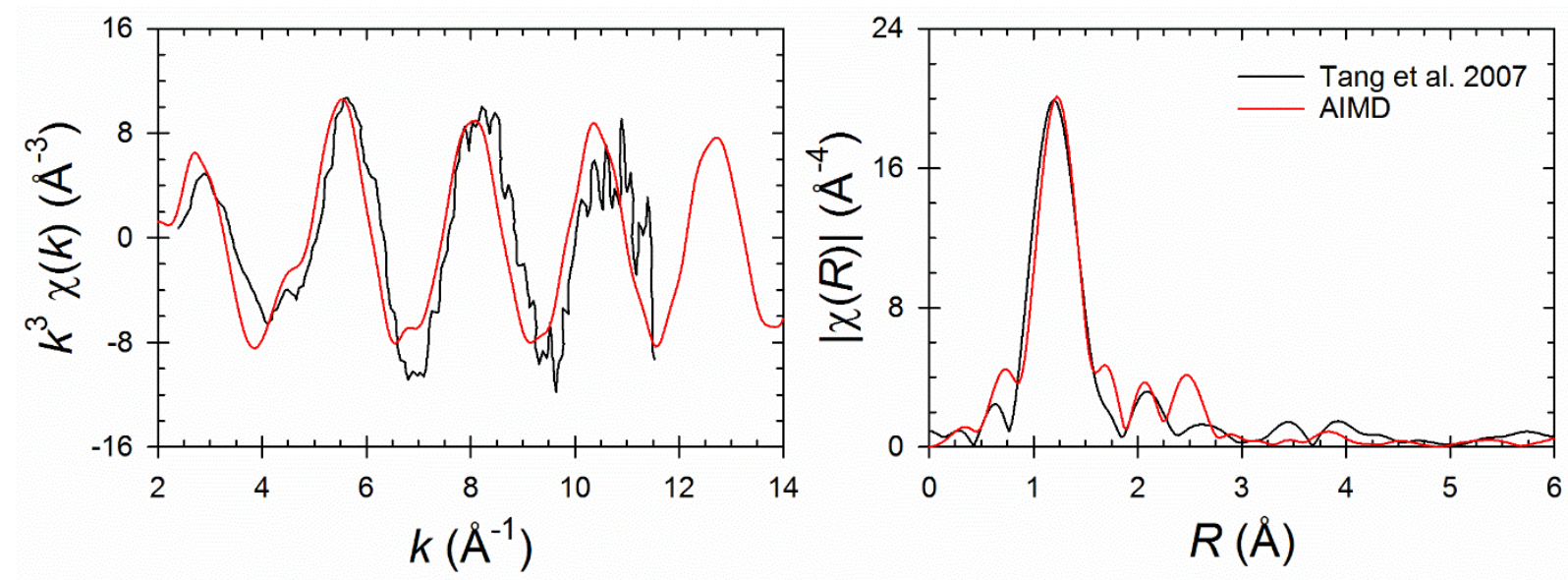

Figure S3. Experimental ${ }^{15}$ and calculated Cr K-edge EXAFS spectra (left) and corresponding Fourier transform magnitudes (right; Hanning window, $\mathrm{d} k=1 \AA^{-1}, 2.5 \AA^{-1} \leq k \leq 11.5 \AA^{-1}$ ) for $\mathrm{CrO}_{4}{ }^{2-}$ in calcite at room temperature (experimental) and $298 \mathrm{~K}$ (calculated).
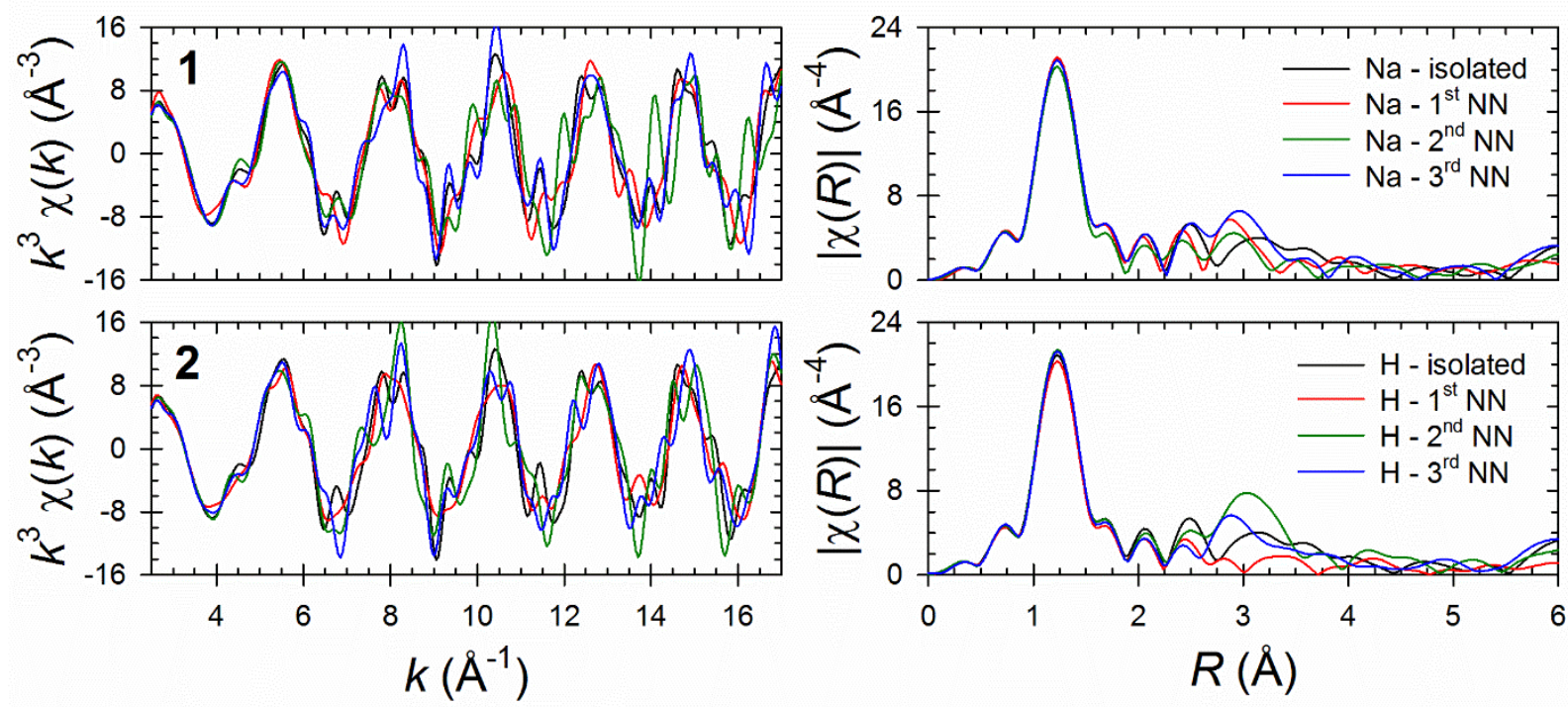

Figure S4. Calculated Cr K-edge EXAFS spectra (left, $8 \mathrm{~K}$ ) and corresponding Fourier transform magnitudes (right; Hanning window, $\mathrm{d} k=1 \AA^{-1}, 2.5 \AA^{-1} \leq k \leq 11.5 \AA^{-1}$ ) for $\mathrm{CrO}_{4}{ }^{2-}$ and $\mathrm{IO}_{3}{ }^{-}$co-incorporated in calcite with the chromate ion in the first, second, or third nearestneighbor (NN) position around iodate and either $\mathrm{Na}^{+}(1)$ or $\mathrm{H}^{+}(2)$ as the charge compensating species. 

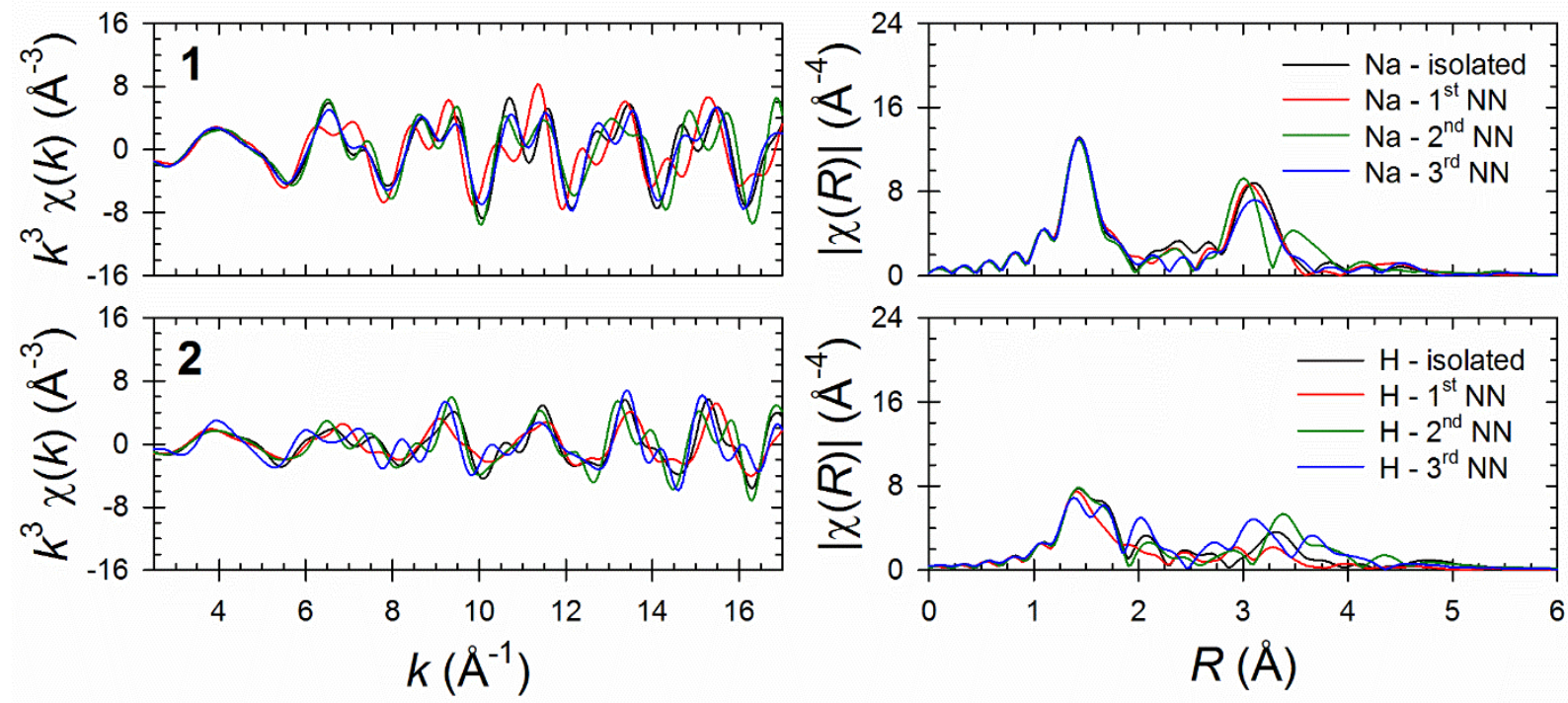

Figure S5. Calculated I K-edge EXAFS spectra (left, $8 \mathrm{~K}$ ) and corresponding Fourier transform magnitudes (right; Hanning window, $\mathrm{d} k=1 \AA^{-1}, 3.5 \AA^{-1} \leq k \leq 16.3 \AA^{-1}$ ) for $\mathrm{CrO}_{4}{ }^{2-}$ and $\mathrm{IO}_{3}{ }^{-}$co-incorporated in calcite with the chromate ion in the first, second, or third nearestneighbor (NN) position around iodate and either $\mathrm{Na}^{+}(1) \mathrm{or} \mathrm{H}^{+}(2)$ as the charge compensating species 


\section{References}

1. Kresse, G.; Hafner, J., Ab initio molecular dynamics for liquid metals. Physical Review $B$ 1993, 47, 558-561.

2. Kresse, G.; Hafner, J., Ab initio molecular-dynamics simulation of the liquid-metalamorphous-semiconductor transition germanium. Physical Review B 1994, 49, 14251-14269.

3. Kresse, G.; Furthmuller, J., Efficient iterative schemes for ab initio total-energy calculations using a plane-wave basis set. Physical Review B 1996, 54, 11169-11186.

4. Kresse, G.; Furthmuller, J., Efficiency of ab-initio total energy calculations for metals and semiconductors using a plane-wave basis set. Comput. Mater. Sci. 1996, 6, 15-50.

5. $\quad$ Blöchl, P. E., Projector augmented-wave method. Physical Review B 1994, 50, 17953 17979.

6. Kresse, G.; Joubert, D., From ultrasoft pseudopotentials to the projector augmented-wave method. Physical Review B 1999, 59, 1758-1775.

7. $\quad$ Perdew, J. P.; Burke, K.; Ernzerhof, M., Generalized gradient approximation made simple. Physical Review Letters 1996, 77 (18), 3865-3868.

8. $\quad$ Perdew, J. P.; Burke, K.; Ernzerhof, M., Generalized gradient approximation made simple [Phys. Rev. Lett. 77, 3865 (1996)]. Physical Review Letters 1997, 78 (7), 1396-1396.

9. Grimme, S., Semiempirical GGA-type density functional constructed with a long-range dispersion correction. Journal of Computational Chemistry 2006, 27, 1787-1799.

10. Kerisit, S. N.; Smith, F. N.; Saslow, S. A.; Hoover, M. E.; Lawter, A. R.; Qafoku, N. P., Incorporation Modes of Iodate in Calcite. Environmental Science \& Technology 2018, 52 (10), 5902-5910.

11. Rehr, J. J.; Albers, R. C., Theoretical approaches to X-ray absorption fine structure. Rev. Mod. Phys. 2000, 72, 621.

12. Rehr, J. J.; Kas, J. J.; Prange, M. P.; Sorini, A. P.; Takimoto, Y.; Vila, F. D., Ab initio theory and calculations of X-ray spectra. C.R. Phys. 2009, 10 (6), 548-559.

13. Rehr, J. J.; Kas, J. J.; Vila, F. D.; Prange, M. P.; Jorissen, K., Parameter-free calculations of x-ray spectra with FEFF9. Phys. Chem. Chem. Phys. 2010, 12, 5503-5513.

14. Newville, M., IFEFFIT: Interactive XAFS analysis and FEFF fitting. Journal of Synchrotron Radiation 2001, 8, 322-324.

15. Tang, Y.; Elzinga, E. J.; Lee, Y. J.; Reeder, R. J., Coprecipitation of chromate with calcite: Batch experiments and X-ray absorption spectroscopy. Geochim. Cosmochim. Acta 2007, 71, 1480-1493. 